
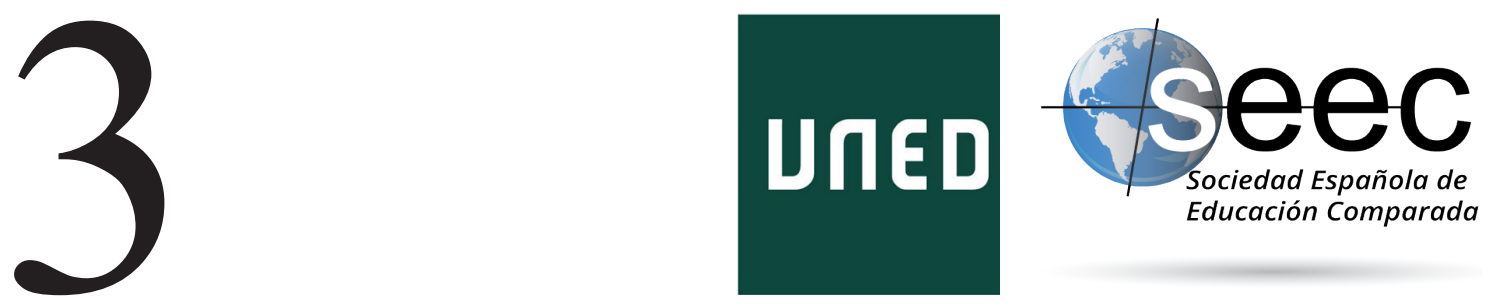

\title{
El «statu quo»y las perspectivas del programa de movilidad internacional de estudiantes chinos con las universidades españolas
}

\author{
The "Statu quo" and Perspectives of the \\ International Mobility Program of Chinese \\ Students with Spanish Universities
}

\section{Chen Xing*; Li Zixin**}

DOI: $10.5944 /$ reec.34.2019.23620

\section{Recibido: 28 de enero de 2019 Aceptado: 21 de junio de 2019}

*Chen Xing: Profesor titular del Departamento de Filología Hispánica de la Universidad de Estudios Extranjeros de Guangdong, Guangzhou, República Popular China y doctorando del Centro de Literatura y Cultura Extranjeras de la misma universidad. Está especializado en la comunicación intercultural y pedagogía. Datos de contacto: E-mail: chenxing@gdufs.edu.cn

**Li Zixin: Estudiante del Departamento de Filología Hispánica de la Universidad de Estudios Extranjeros de Guangdong, Guangzhou, República Popular China. Datos de contacto: E-mail: 963507553@qq.com 


\title{
Resumen
}

En el contexto de la globalización e internacionalización de la educación superior, la movilidad internacional de estudiantes se ha convertido cada vez más en una estrategia importante para que los países participen en la competencia internacional de desarrollo. Los intercambios entre universidades a nivel internacional se están reforzando cada día más, y el proyecto de intercambio estudiantil es una de las formas más importantes de cooperación. Los estudiantes universitarios de idiomas extranjeros siempre han sido y son más activos en la movilidad debido a su especial naturaleza académica. Este artículo toma la especialidad de filología hispánica en China como ejemplo para ilustrar la situación actual de sus estudiantes en el programa de intercambio con las universidades españolas. A través de encuestas, cuestionarios y método de análisis estadístico SPSS, se analizan los problemas existentes en el actual marco de programa y la correlación entre variables, y se proponen contramedidas y sugerencias correspondientes con miras a un modelo internacional de cooperación e intercambio universitario más amplio y connotativo en la nueva situación.

Palabras clave: programa de movilidad internacional de estudiantes; intercambio de estudiantes; estudiantes chinos de español; universidades españolas

\begin{abstract}
In the context of globalization and internationalization of higher education, increasing international students' exchange has become an important strategy for countries to participate in international development competition. The exchanges between international universities are enormously strengthened, among which the student exchange program is one of the most important forms of cooperation. What's more, students of foreign language universities have always been particularly active in these intercultural exchanges due to their special academic nature. This article takes Chinese students of Spanish major as an example to illustrate the current situation of Chinese students in the exchange programs with Spanish universities. This paper uses various research methods such as surveys, questionnaires and SPSS Statistics, with the aim of reviewing the problems existing in the current exchange programs and analyzing the relations between some certain variables, which helps us put forward corresponding measures and suggestions for further development and look into a broader and richer future of the exchanges and cooperation between universities worldwide in the new situation.
\end{abstract}

Key Words: International Exchange Program of students; student exchange; Chinese students of Spanish major; Spanish universities 


\section{Introducción}

A partir de la década de 1990, especialmente después de la entrada del siglo XXI, bajo la influencia de la globalización económica, la educación superior apuesta por elevar su nivel de internacionalización y globalización. El núcleo de su internacionalización es que las universidades modernas abren sus puertas hacia el mundo con la misión de formar la conciencia integral y globalizada, y la capacidad para tomar parte en la competencia internacional. Siguiendo esta tendencia, el Ministerio de Educación chino propone en su Plan Nacional de Reforma y Desarrollo de la Educación a Mediano y Largo Plazo ampliar la apertura de la educación, fortalecer los intercambios y cooperación internacionales, introducir recursos educativos de calidad y mejorar el nivel de los intercambios y cooperación, con el objetivo de cultivar gran número de talentos con visión internacional, grandes conocedores de las normas internacionales y capaces de participar en asuntos y competencias internacionales. Para lograr el objetivo, el Plan alienta programas de intercambio internacional en las universidades chinas apoyando el intercambio de estudiantes entre universidades chinas y extranjeras, y el reconocimiento mutuo de créditos y títulos e innovando y mejorando el mecanismo de seleccionar y becar a estudiantes sobresalientes para que estudien en universidades e instituciones extranjeras de investigación. Hoy en día, el modelo de programas de movilidad internacional de estudiantes se ha convertido en una de las formas más importantes para promover intercambios y colaboración entre universidades, optimizar la distribución de recursos educativos, reformar el modo de formar talentos internacionalizados, mejorar la competencia internacional de los estudiantes y elevar el poder blando cultural del país.

\section{Marco teórico}

De acuerdo con Cordera y Santamaría (2008), la internacionalización de la educación superior comprende cuatro principales modalidades: internacionalización individual, internacionalización institucional, internalización regional, subregional y entre regiones y proveedores externos de educación superior. La movilidad estudiantil forma parte de la internacionalización de la educación superior en las modalidades individual y regional. En la primera modalidad, la movilidad estudiantil se lleva a cabo de forma independiente o como parte de un programa específico, con financiamiento o sin él, mientras que en la segunda modalidad se implementan programas multilaterales de aprendizaje de idiomas, movilidad académica y vinculación de las universidades con el sector empresarial (Palma, 2013). Con el paso de que la educación superior apuesta por mejorar su nivel de internacionalización y globalización, hoy en día, la movilidad de estudiantes universitarios es la principal faceta de la internacionalización de la educación superior. El proceso de internacionalización comprende y se nutre de una gama de actividades muy amplia, que inciden de manera directa o indirecta en la circulación internacional de estudiantes. Una de esas actividades es la provisión de servicios de educación superior en el extranjero (Luchilo, 2006).

El proceso de Bolonia se inició en 1999 con el fin de hacer más competitivo y atractivo al sistema educativo europeo y luego dio origen a programas de colaboración con otras regiones, como el proyecto Erasmus Mundus. De los diez objetivos establecidos en la Declaración de Bolonia la mayor parte de ellos están destinados a eliminar las barreras a la movilidad estudiantil, y los otros cuatro son para que el sistema europeo de enseñanza 
superior adquiera un grado de atracción mediante la movilidad estudiantil (fecha de consulta: 20/06/2019). Más tarde, el Comunicado de Lovaina-la Nueva de 2009 estableció claramente los objetivos específicos de la movilidad de los estudiantes: la movilidad será el sello distintivo del Espacio Europeo de Educación Superior y en el año 2020, al menos un 20 \% de aquellos que se titulen dentro del Espacio Europeo de Educación Superior deben haber disfrutado de un período de estudios o de formación en el extranjero (fecha de consulta: 20/06/2019).

En el caso de China, desde la entrada en la OMC (Organización Mundial del Comercio), el Estado ha elaborado una serie de políticas educativas, entre las cuales se destacan Plan Nacional de Reforma y Desarrollo de la Educación a Mediano y Largo Plazo (20102020) promulgado en 2010, Resoluciones de la Apertura de la Educación en la Nueva Era emitidas en 2016 y Campaña Educativa bajo "la Franja y la Ruta" publicada en 2016. Sin embargo, el Gobierno no ha dado instrucciones claras sobre la garantía de la calidad y las medidas específicas para promover la internacionalización de la educación superior. A partir de 2015, la Asociación de Intercambio Internacional de Educación de China, encargada por el Ministerio de Educación, realiza anualmente una encuesta sobre la internacionalización de las universidades, cuyos indicadores de evaluación se consideran como directrices nacionales de evaluación para la internacionalización de la educación superior ( $\mathrm{Wu}, 2019)$. Cabe mencionar que el nivel de la internacionalización de la especialidad universitaria de español no ha llamado atención de dicha Asociación.

\section{Status quo de la cooperación internacional de la especialidad de español en China}

A partir del 1952, año en que se fundó el primer centro de enseñanza de español en la Universidad de Estudios Extranjeros de Beijing, el número de departamentos de español ha llegado al 91 con más de 16.000 alumnos de grado ${ }^{1}$. En los 60 años transcurridos, a medida que la reforma y apertura de China y la globalización económica, la especialidad de español, junto con todas las otras especialidades ha experimentado una serie de ajustes, tales como rediseñar el plan curricular y reformular el objetivo de la formación de estudiantes, con el fin de adaptarse a los cambios y la demanda del mercado laboral. Según Lu Jingsheng (2018), director del Departamento de Español del Comité de Orientación para la Enseñanza de Lenguas Extranjeras del Ministerio de Educación Chino y profesor catedrático de la Universidad de Estudios Internacionales de Shanghái, en respuesta a la situación actual las universidades deberían adoptar un modelo innovador e integral de capacitación de talentos combinando la enseñanza de lenguas extranjeras con otras especialidades profesionales para formar estudiantes multilingües con visión internacional que satisfagan las necesidades del desarrollo social ${ }^{2}$.

Para conocer el status quo de la cooperación internacional de la especialidad de español en China, hemos realizado investigaciones en algunas universidades del país. Teniendo en consideración factores como la duración del departamento, la categoría

1 Cifra publicada en el Congreso Anual de los Departamentos de Español de las Universidades de China (Año 2018), que tuvo lugar en Heilongjiang, China.

2 Conferencia plenaria dictada por Lu Jingsheng en el Simposio Internacional de la Enseñanza de Español en China, que tuvo lugar de 18 a 20 de septiembre de 2018 en la Universidad de Estudios Internacionales de Shanghái. Noticia publicada en la página oficial de web de la universidad: http://wmcj.shisu.edu.cn/ b6/da/c990a112346/page.htm (Fecha de consulta: 20-06-2019) 
de la universidad, la característica geográfica de la universidad, etc., intentamos proporcionar una representación más completa y objetiva del nivel y el estado de la cooperación internacional de la especialidad de español con los objetos de encuesta ${ }^{3}$ que hemos seleccionado.

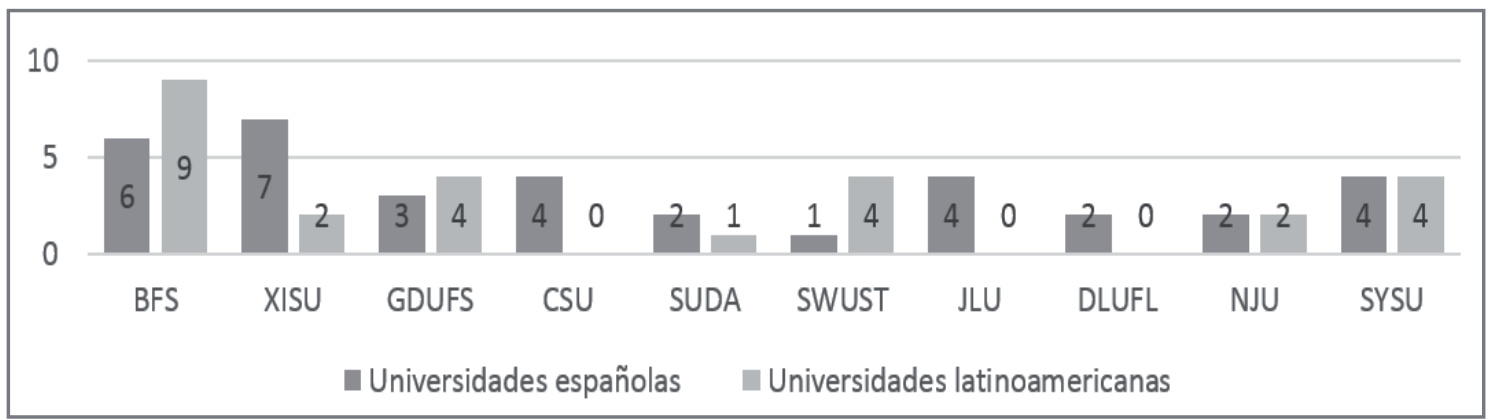

Figura 1. Número de programas de movilidad con las universidades españolas y latinoamericanas

A través de la encuesta, hemos encontrado que todas las universidades promueven activamente el proceso de internacionalización, y que los profesores, el personal de administración y los estudiantes son beneficiarios de la internacionalización, y que los programas de intercambio estudiantil son uno de los medios más tradicionales y relativamente mejor desarrollados para cultivar talentos internacionalizados. Estos programas se basan en acuerdos firmados por los socios institucionales, por los que se intercambian alumnos en un cierto período de tiempo. Cabe destacar que dichos programas no implican la concesión del título sino el reconocimiento mutuo de los créditos cursados durante el período del intercambio. Aproximadamente a principios del siglo XXI los departamentos de español en China empezaron este tipo de programas. Los que tengan el establecimiento temprano, una buena base para el desarrollo y una buena estructura docente no escatiman esfuerzos en la cooperación internacional manteniendo una colaboración estrecha y estable con universidades españolas y latinoamericanas a través de la cual proporcionan a los estudiantes una gran cantidad de recursos educativos internacionales de alta calidad. En los últimos años el porcentaje de los estudiantes de intercambios en estos departamentos ha llegado al más de $50 \%$. Y los departamentos fundados en los últimos diez años sientan su base, desde el principio, en la formación de talentos con gran competencia intercultural mediante la cooperación con las universidades de países hispanohablantes. Cabe señalar que en algunos centros de enseñanza todos los estudiantes del tercer año son enviados a las universidades extranjeras para hacer el intercambio de estudios, y de esta manera se alivia efectivamente la presión generada por la escasez de docentes.

El hecho de que la mayoría de los estudiantes de intercambio internacional sean del tercer año tiene sus explicaciones. Por un lado, debido a los diferentes sistemas educativos y peculiaridades de la especialidad, ninguna universidad de los países hispanohablantes puede ofrecer un plan curricular igual que el de sus universidades de origen,

3 Universidad de Estudios Extranjeros de Beijing (BFS, 1952), Universidad de Estudios Internacionales de $\mathrm{Xi}^{\prime}$ an (XISU, 1965), Universidad de Estudios Extranjeros de Guangdong (GDUFS, 1965), Universidad Central del Sur (CSU, 2011), Universidad de Soochow (SUDA, 2010), Universidad de Ciencia y Tecnología del Suroeste (SWUST, 2008), Universidad de Jilin (JLU, 2000), Universidad de Estudios Internacionales de Dalian (DLUFL, 2002), Universidad de Nanjing (NJU, 1964) y Universidad de Sun Yat-sen (SYSU, 2009) 
ya que la filología hispánica en China en realidad implica el aprendizaje de una lengua extranjera y su cultura desde cero mientras que en los países de lengua hispana el idioma no es más que una herramienta para aprender lo relacionado con él mismo. De modo que una base lingüística sólida es indispensable para lograr el objetivo del intercambio. Por el otro, los estudiantes chinos del segundo año tienen previsto el examen nacional de español (examen elemental de español, conocido como EEE4 y reconocido a nivel nacional) y los del cuarto, preocupados por su salida profesional, también tienen otro examen de nivel superior y trabajo final del grado. Por lo cual, los alumnos del tercer año son candidatos ideales para participar en los programas de movilidad, lingüísticamente fáciles de adaptarse al entorno extranjero y competentes para asignaturas de carácter académico y profesional en español. Todos los estudiantes de intercambio están exentos de la matrícula de la universidad de acogida y algunos de ellos son becados por los programas del Consejo de Becas de China o por alguna institución patrocinadora extranjera.

El gobierno español y las universidades españolas son más solidarios en la promoción de la internacionalización educacional. En los últimos cinco años, el Ministerio de Educación Español, la Embajada Española y la Oficina Económica y Comercial Española en China han organizado sucesivamente Exposición de Educación de España y uno de cuyos objetivos es traer delegaciones universitarias en busca de colaboraciones con universidades chinas.

Como hemos visto, la movilidad internacional de estudiantes es una tendencia irreversible. Su desarrollo en China ha llegado a otra etapa, en la que el mayor número de instituciones cooperativas deja de ser la meta y la calidad de programas de intercambio se convierte en el elemento decisivo de hacer posible la colaboración interuniversitaria. Basándose en el principio de dar prioridad al desarrollo estudiantil el ranking de la universidad y de la especialidad, la calidad de la docencia y de la administración, el plan curricular son factores más considerados antes de firmar los convenios entre universidades.

\section{Objetivos y diseño de investigación}

El propósito de este trabajo consiste en i) conocer el desarrollo de los programas de movilidad específicamente dirigidos a los alumnos de español y el grado de satisfacción de los estudiantes concernientes; ii) hacer fortalecer la gestión de administración durante todo el período (antes, durante y después) del intercambio estudiantil; iii) conducir los programas hacia un desarrollo saludable, eficaz y propicio tanto para el departamento de español como para sus estudiantes. Debido a la no poca cantidad de países de habla española y las diversidades del currículo disciplinar y del estándar de crédito entre ellos, para facilitar la investigación hemos tomado como objetos los programas acordados con las universidades españolas, los cuales se caracterizan por la alta gama y la larga historia de cooperación y el desarrollo más sistematizado. De esta manera, en el contexto del mismo sistema de educación superior español, nos conviene dar con las características y diferencias de la cooperación con diferentes instituciones españolas, y las diferencias en los modelos y efectos de cooperación entre instituciones chinas y españolas. No cabe duda de que los resultados de esta investigación tienen referencia a los programas elaborados con las universidades latinoamericanas.

Mediante la plataforma en línea Wenjuanxing diseñamos un cuestionario titulado «Investigación sobre los programas de movilidad con las universidades españolas dirigidos a los estudiantes chinos», el cual está compuesto por cuatro partes: datos personales, 
preparativos para el intercambio, período del intercambio y efecto del intercambio. En la primera parte se recopilan informaciones como las universidades de origen y de acogida, año en que cursan, plazo del intercambio y fondos o ayuda económica que reciben, etc.; en la segunda parte se intenta conocer el mecanismo de la selección de estudiantes para el intercambio, los motivos de elegir la institución de acogida y las orientaciones al elegir asignaturas, etc.; Durante el proceso del intercambio se presta atención a los trabajos orientativos hechos por la universidad de acogida y sus efectos, los estudios y la vida del estudiante de intercambio, etc.; por último, se intenta conocer la evaluación del programa de movilidad por parte del estudiante y la auto-evaluación de sus estudios durante la estancia.

Hasta el día 1 de noviembre de 2018, se habían recopilado 219 cuestionarios. Por medio del conocimiento estadístico y el software SPSS intentamos analizar el grado de satisfacción de los estudiantes respecto a los programas de intercambios con las universidades españolas y los factores que determinan sus evaluaciones, los defectos de los programas existentes, y después se proponen sugerencias.

\section{Análisis del cuestionario}

\subsection{Corpus del cuestionario}

El cuestionario había sido contestado por 219 estudiantes provenientes de una veintena de universidades chinas, distribuidas por todas partes del país, por ejemplo, Helongjiang, Beijing, Xi'an, Shanghái, Hunan, Zhejiang, Jiangsu, Sichuan, Guangdong, etc. Los estudiantes hicieron sus estudios de intercambio principalmente en el tercer año del grado (92,24\%) en más de quince universidades españolas respectivamente durante 4-6 meses (23,29 \%), 7-9 meses (26,94\%) o 10-12 meses (49,32 \%), y los programas son de carácter i) convenios interuniversitarios (69,86 \%); ii) proyectos del Consejo Nacional de Becas de China (10,5 \%); iii) programas ERASMUS (8,68 \%) y iv) otros (10,96 \%).

\subsection{Grados de satisfacción y factores determinantes}

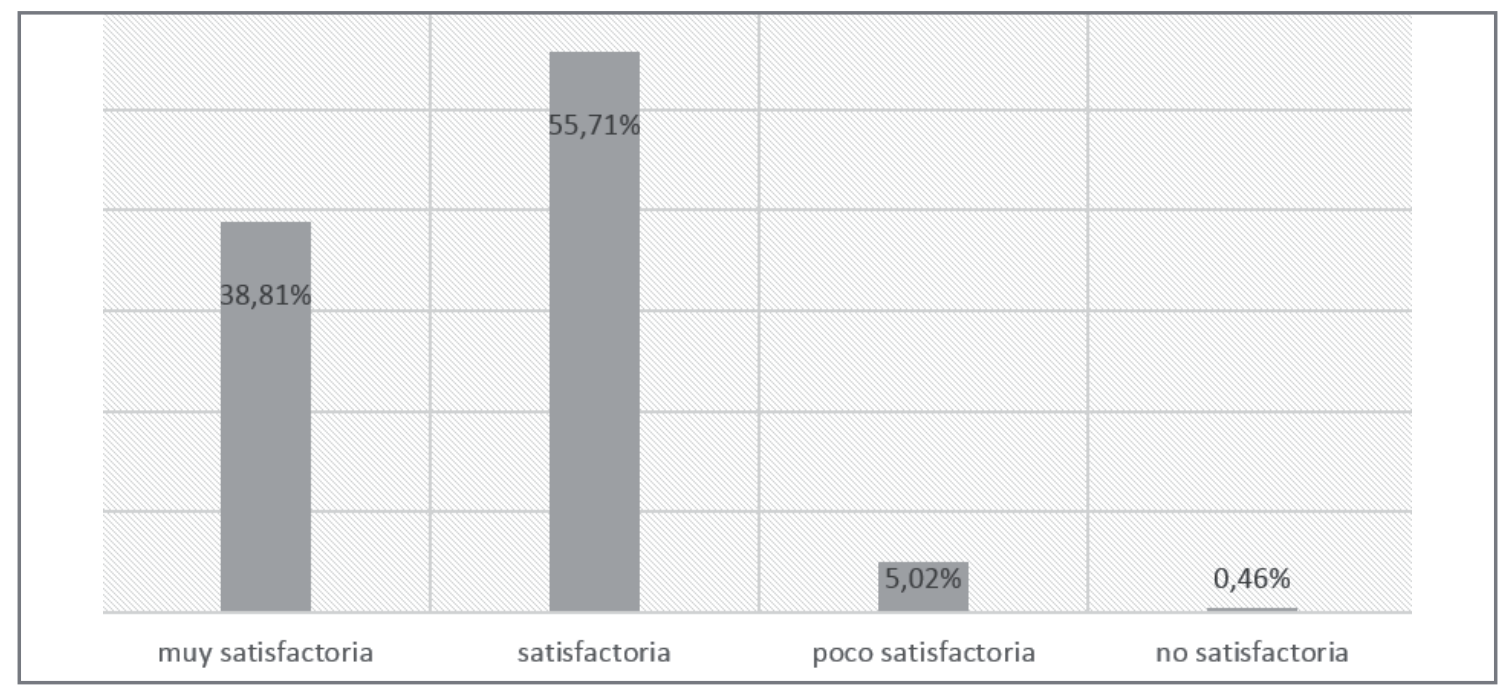

Figura 2. Evaluación general del programa de movilidad internacional 
De acuerdo con los resultados del ítem «Su evaluación general del programa de movilidad internacional» (Figura 2), se puede observar que más del 94 \% de la evaluación por parte de los estudiantes puede describirse como «satisfactoria» mientras que unos pocos se sintieron «no muy satisfechos» y un estudiante demostró su desilusión con el programa. Y entre los que se mostraron satisfechos, más estudiantes tienden a la opción «satisfactoria» en lugar de «muy satisfactoria».

En base del concepto de que el efecto del programa está estrechamente vinculado con la evaluación de los estudiantes, combinamos el coeficiente de correlación de Pearson con varios variables en el proceso del intercambio (curso académico del intercambio, duración, formación previa a la partida, los obstáculos lingüísticos en la vida y en el estudio, oferta de asignaturas en las universidades españolas, etc.) con el objetivo de sacar los factores que tengan una correlación positiva significativa con el grado de satisfacción de los estudiantes.

Según el análisis de correlación de Pearson, los tres factores más influyentes relacionados con la evaluación del programa del intercambio por parte de los estudiantes son «enfoque docente y forma de evaluación de las universidades españolas», «obstáculos en los estudios durante el intercambio» $\mathrm{y}$ «barreras en la vida durante el intercambio».

\begin{tabular}{|c|c|c|}
\hline \multicolumn{3}{|c|}{ Coeficiente de correlación Pearson } \\
\hline & & $\begin{array}{l}\text { Su evaluación general del programa de } \\
\text { movilidad internacional }\end{array}$ \\
\hline \multirow{2}{*}{ Barreras lingüísticas en la vida cotidiana } & coeficiente de correlación & $0,224^{* *}$ \\
\hline & Valor $p$ & 0,006 \\
\hline \multirow{2}{*}{ Barreras lingïísticas en los estudios } & coeficiente de correlación & $0,274^{* *}$ \\
\hline & Valor $\mathrm{p}$ & 0,001 \\
\hline \multirow{2}{*}{ Reconocimiento de créditos cursados en España } & coeficiente de correlación & $0,008^{* *}$ \\
\hline & Valor $\mathrm{p}$ & 0,922 \\
\hline \multirow{2}{*}{ Adaptación a la docencia y evaluación en España } & coeficiente de correlación & $0,351^{* *}$ \\
\hline & Valor $\mathrm{p}$ & 0,000 \\
\hline
\end{tabular}

Figura 3. Coeficiente de correlación Pearson

Como se puede ver en la Figura 3, el coeficiente de correlación entre «Evaluación general del programa de intercambio con las universidades españolas» $\mathrm{y}$ «Dificultades lingüísticas en la vida cotidiana» es de 0,224 y muestra un nivel significativo de o,o1, lo que indica que existe una correlación positiva significativa entre estas dos variables. El coeficiente de correlación entre «Evaluación general del programa de intercambio con las universidades españolas» $\mathrm{y}$ «Dificultades lingüísticas en los estudios» es de 0,274 y muestra un nivel significativo de 0,01, lo que supone también una correlación positiva significativa entre las dos variables. En cuanto a las dificultades lingüísticas en la vida cotidiana y en los estudios, se muestra cierta diferencia entre estudiantes de diferentes cursos: los estudiantes de primer y segundo cursos se encuentran con más dificultades en la vida cotidiana que en los estudios mientras que los resultados de los alumnos de tercer y cuarto cursos son contrarios, la cual se debe en cierto sentido a la oferta de asignaturas de las universidades españolas: a los alumnos que tengan el nivel lingüístico más bajo se ofrecen asignaturas más relacionadas con la competencia lingüística y a los alumnos 
mejor habilitados lingüísticamente, asignaturas de carácter más académico y profesional. El valor de coeficiente de correlación entre «Evaluación general del programa de intercambio con las universidades españolas» y «Adaptación al enfoque docente y la forma de evaluación de las universidades españolas» 0,351 y un nivel significativo de o,01 significan una correlación positiva significativa entre las dos variables.

No existe correlación positiva obvia entre factores como curso académico del estudiante, duración del intercambio, formación previa a la partida y la evaluación general del programa por parte de los estudiantes.

Cabe mencionar que el coeficiente de correlación entre «Evaluación general del programa de intercambio con las universidades españolas» y «Reconocimiento de los créditos cursados en España» es de 0,008 y el valor del índice $p$ es de 0,922 (que es mayor que 0,05), lo que indica la inexistencia de una correlación entre los dos ítems. El hecho de que $97 \%$ de los encuestados lograran el reconocimiento de créditos con éxito muestra que este tema, preocupado por las universidades chinas, no es un factor relevante que afecta la evaluación por parte de los estudiantes.

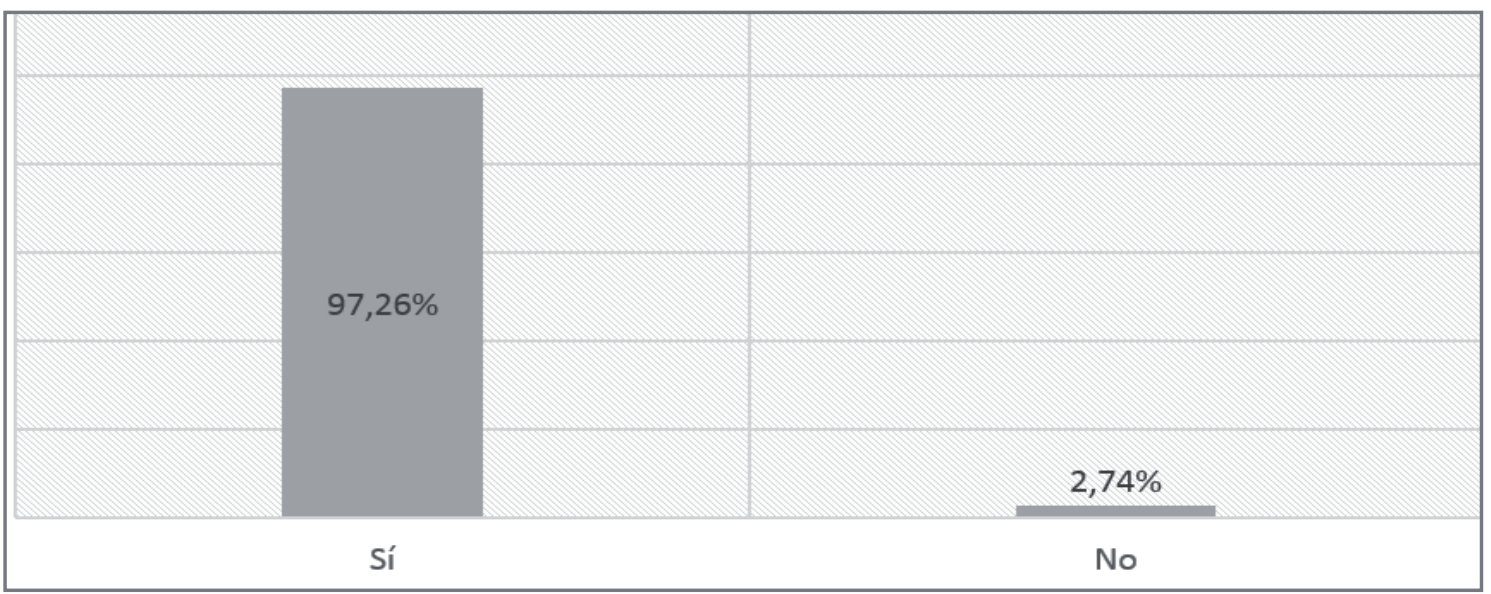

Figura 4. Reconocimiento de créditos cursados en España

\subsection{Problemas existentes en los programas de movilidad internacional}

\section{5•3.1 Falta de mecanismo de administración sistemático y profesional}

En efecto, los departamentos de cooperación internacional y de educación y el departamento de español se encargan de los trabajos preparativos previos a la partida del intercambio, como la solicitud de matrícula en las instituciones de acogida, asesoramiento sobre la visa, formación y orientación al elegir asignaturas, etc. Las instituciones de envío de estudiantes no solo son coordinadoras entre los departamentos interesados dentro de ellas mismas, sino que también son responsables de mantener el constante contacto con las universidades españolas para resolver los problemas en común o de carácter personalizado que enfrentan los estudiantes. Sin embargo, debido al número insuficiente del personal, problema común de muchas universidades chinas, las instituciones de envío caen en el dilema de promover más proyectos buenos o contentarse con los actuales proyectos. En realidad, algunos alumnos no están contentos con los departamentos concernientes al programa de movilidad, por ejemplo, antes de cursar su plazo del intercambio las siete décimas partes de los alumnos tienen muy pocos conocimientos sobre las universidades de acogida y más de la mitad no consigue el currículo disciplinar. Entre los 
El «statu quo» y las perspectivas del programa de movilidad internacional de estudiantes chinos con las universidades españolas

que lo tienen solo la mitad lo consigue por vía oficial de las universidades de envío o de acogida y la otra mitad, por vía internet o alumnos de promociones anteriores.

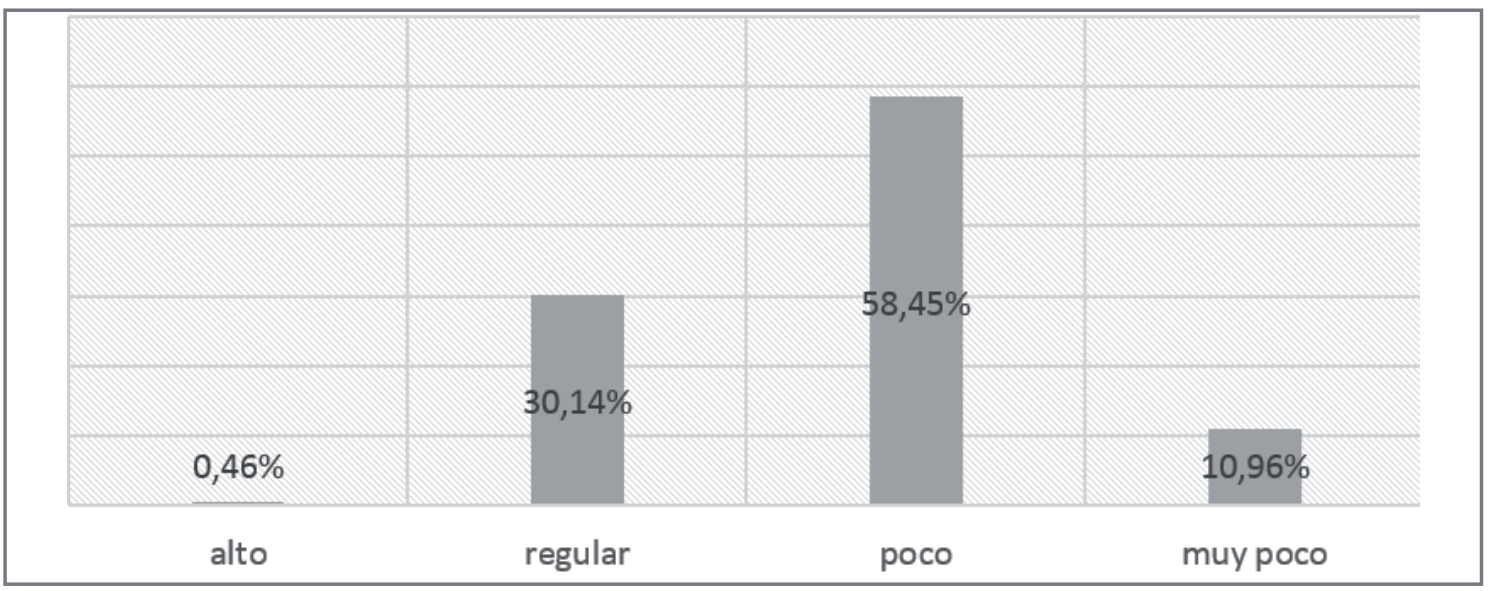

Figura 5. Conocimiento sobre la universidad de acogida antes de la partida

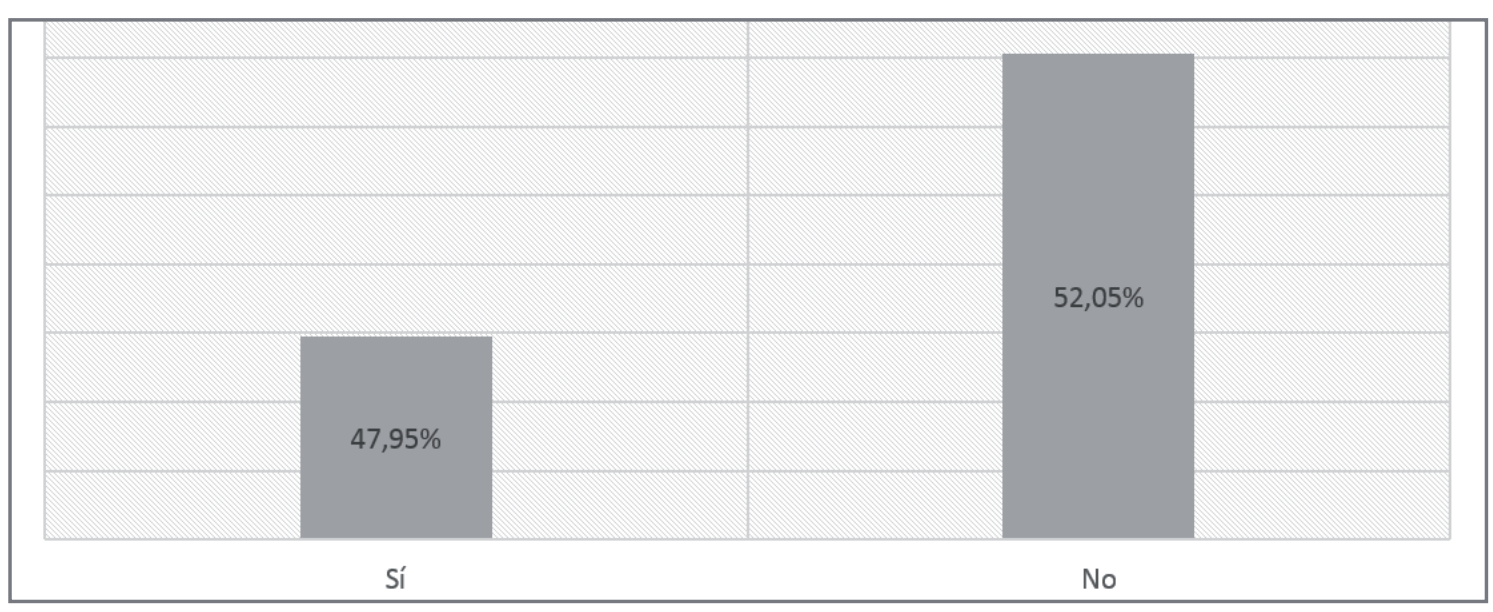

Figura 6. Informaciones sobre el currículo disciplinar y oferta de asignaturas de la universidad de acogida

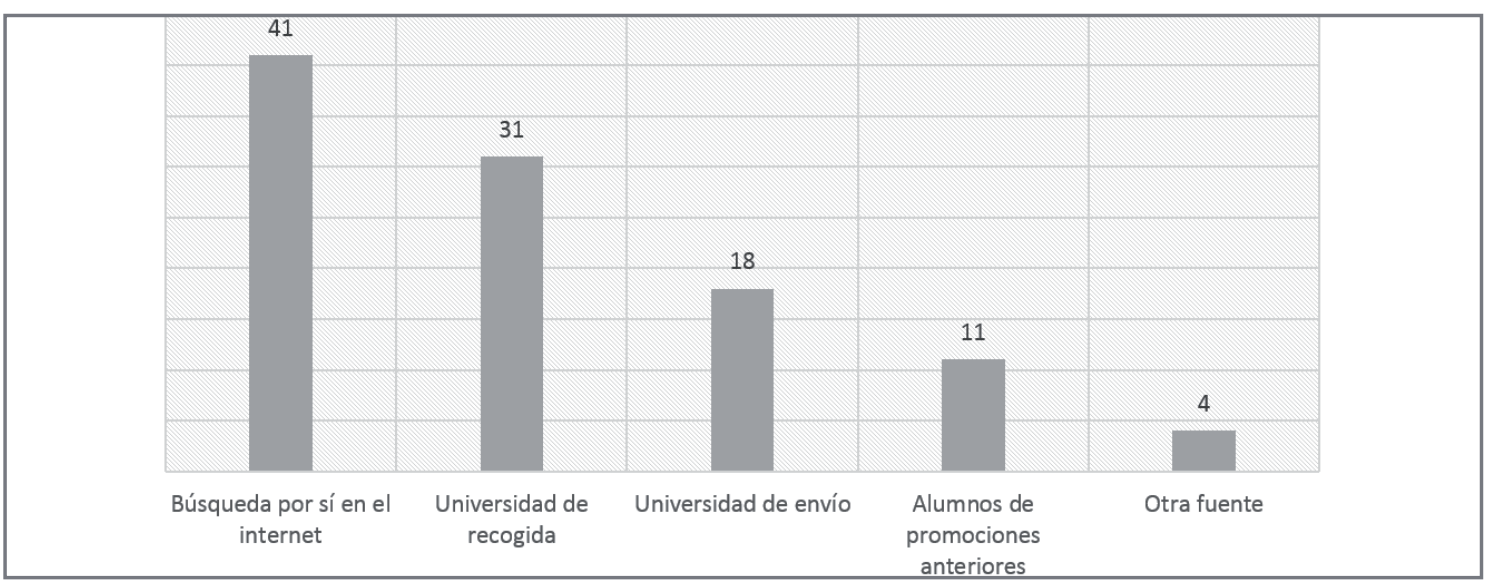

Figura 7. Fuentes de las informaciones sobre el currículo disciplinar y oferta de asignaturas de la universidad de acogida 
A la hora de elegir asignaturas, una dos tercera parte de los encuestados revelaron la inexistencia de formación orientativa en sus universidades de origen, servicio que se figura, de hecho, en la lista de servicios que deben prestar a los alumnos de intercambio. De modo que no es nada extraño que más de $60 \%$ de los estudiantes pidan la fomentación de este servicio para facilitar la adaptación y los estudios en España y el reconocimiento de créditos.

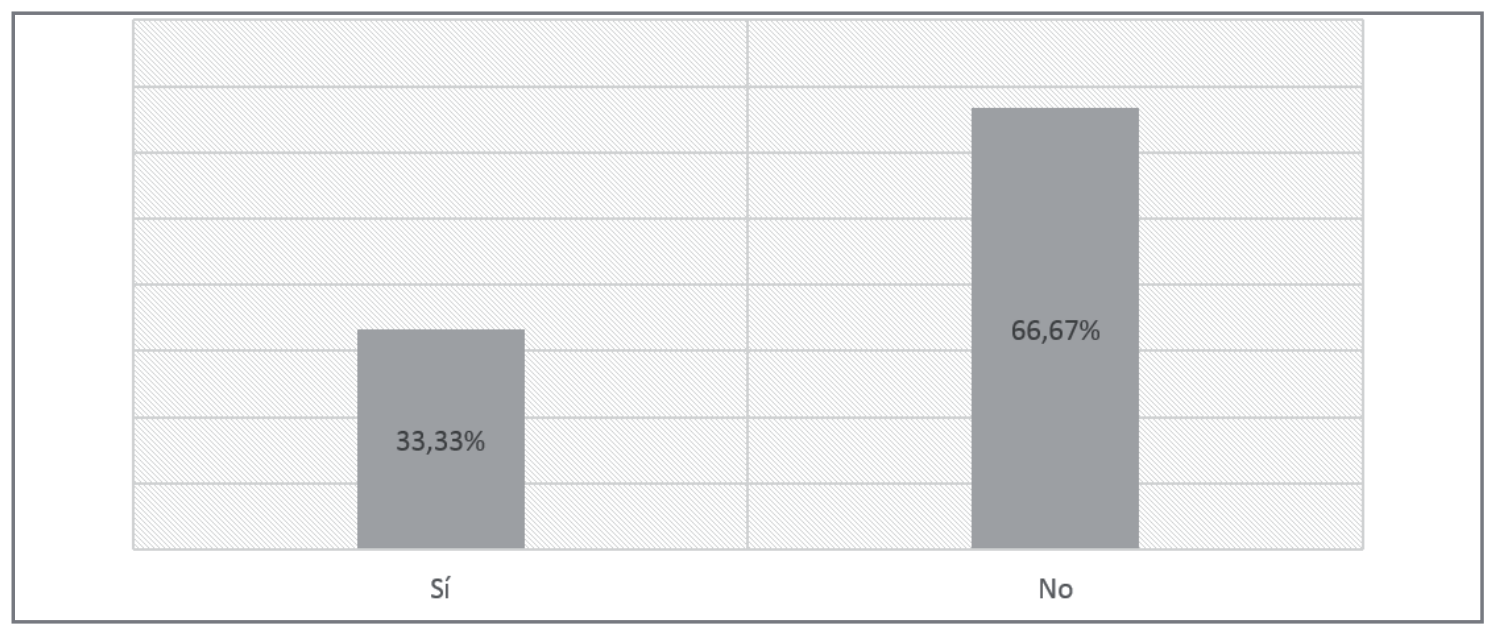

Figura 8. Formación al elegir asignaturas por parte de la universidad de envío

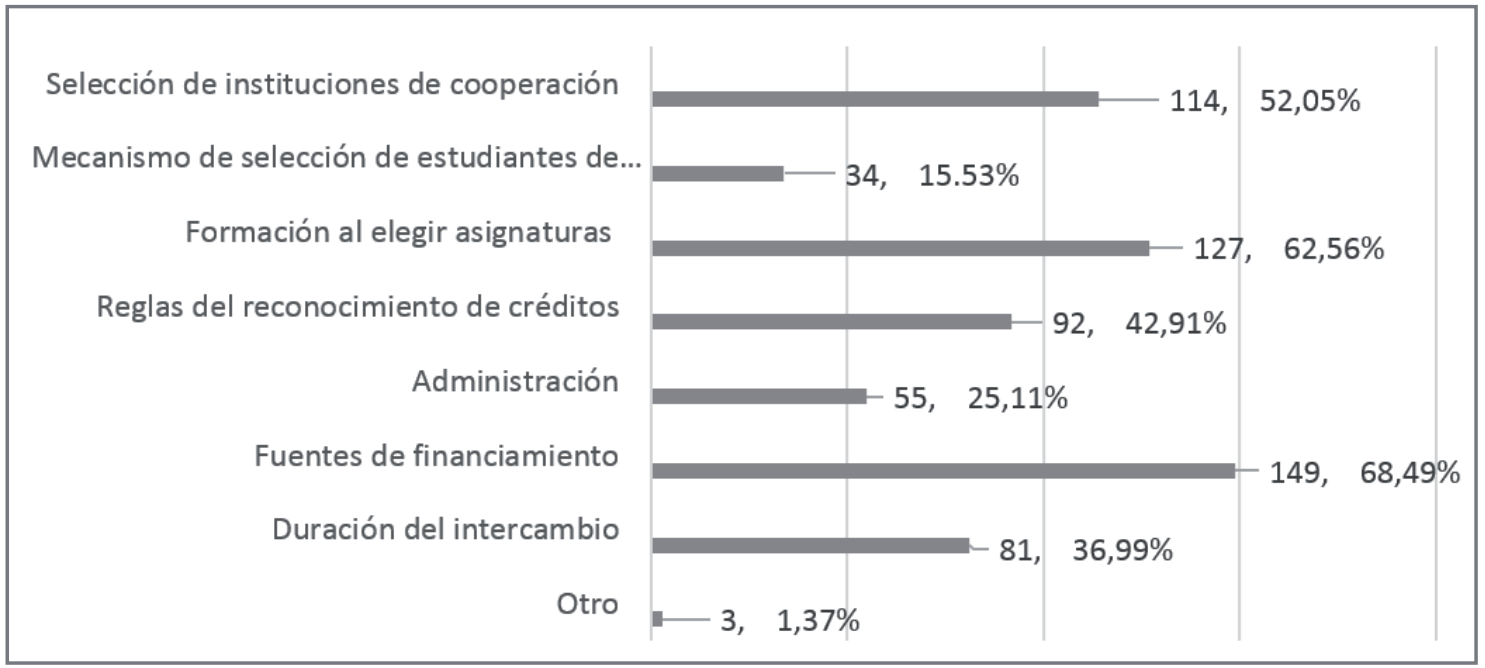

Figura 9. Puntos que se pueden mejorar en los programas de movilidad con España

Además, por la diferencia horaria y la falta de alguna plataforma de comunicación entre universidades la ineficiencia de comunicación hace que los alumnos demoren más tiempo en el papeleo y los trámites.

\section{5•3.2 Difícil adaptación a la vida y los estudios por problemas lingüísticos}

Hay que reconocer que el entorno pragmático y la inserción lingüística son condiciones favorables en los estudios de una lengua extranjera. A los alumnos, a pesar de dos años de estudios de español en China, no les resulta fácil involucrarse en las comunicaciones 
interculturales en el entorno español, e incluso mucho más difícil en los campos académicos y profesionales. Por lo tanto, en el proceso de intercambio, es inevitable que algunos estudiantes permanezcan en silencio en las discusiones grupales debido a las deficientes habilidades de comunicación lingüística. Sin embargo, la participación en clase es un indicador indispensable de la evaluación en España. Según los resultados del cuestionario, la mayoría de los alumnos piensan que las diferencias de la ideología didáctica y las relaciones profesorado-alumnado consisten en que en el sistema educativo español, los alumnos muestran más iniciativas mientras que los profesores, que prestan más atención a la formación de competencia práctica y pragmática, desempeñan un papel complementario. La difícil adaptación hace que algunos estudiantes se sientan desilusionados, e incluso empiecen a tener la idea de volver a China para escaparse de la dura realidad, lo cual es contrario a nuestro propósito original de mejorar sus competencias lingüísticas e interculturales en España.

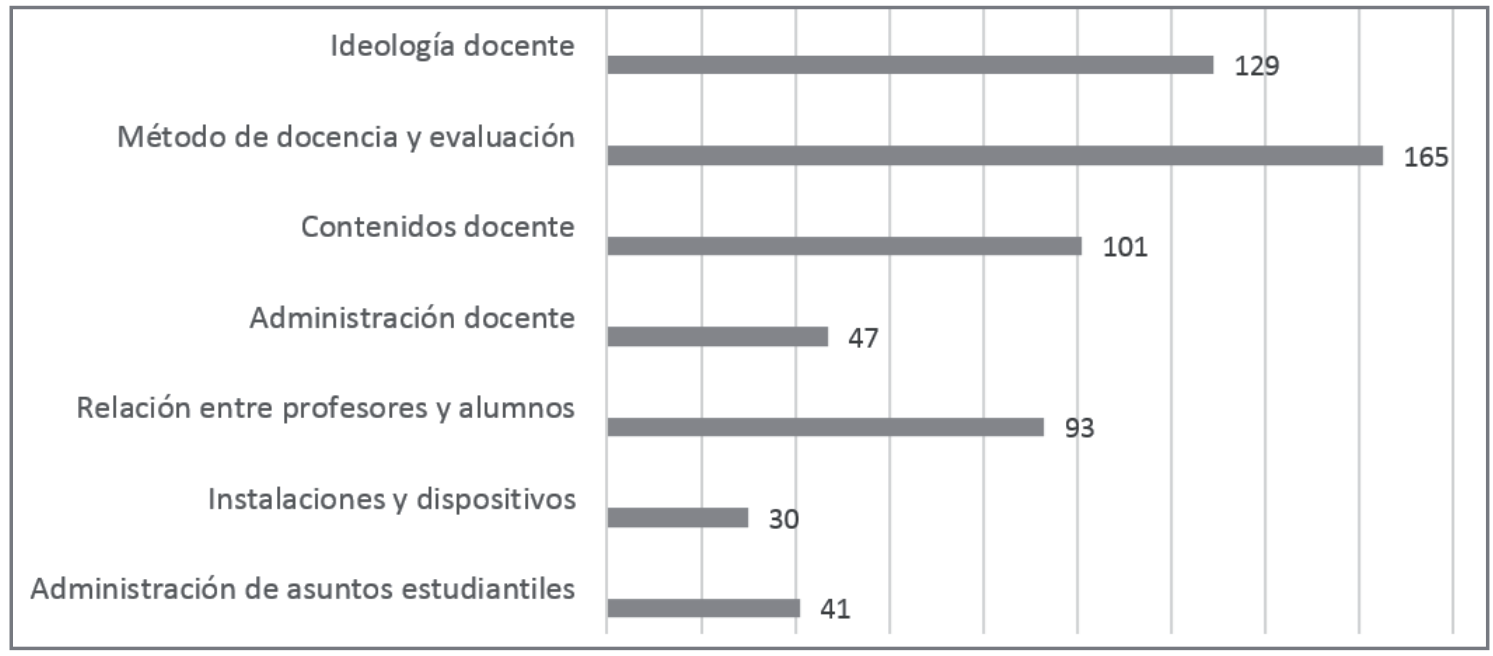

Figura 10. Diferencias entre las universidades chinas y españolas

\subsubsection{Insuficiencia de la ayuda económica}

Los altos gastos también son un elemento que afecta al proceso de intercambio. Según el estándar de financiamiento del Consejo Nacional de Becas de China para estudiantes (2019), el subsidio mensual es de 850 euros por persona ${ }^{4}$ y el Salario Mínimo Interprofesional de España se sitúa actualmente en 900 euros al mes ${ }^{5}$, cifra a la que no llega el nivel promedio chino. Como se ha señalado, la mayoría de estudiantes solo cuentan con la ayuda económica por parte de sus padres sin ningún otro tipo de financiamiento, los gastos suponen una carga no pequeña para una familia común y corriente china. De las sugerencias planteadas por los estudiantes para mejorar los programas de intercambio (véase la Figura 10) se ve que casi el $70 \%$ de estudiantes esperan expandir el canal de becas, lo que muestra el impacto de los factores económicos en el desarrollo de los programas de movilidad.

4 Cifra fijada por el Ministerio de Finanzas y el Ministerio de Educación a través del CKJ[2019]num.6 de 20 de febrero de 2019

$5 \quad$ Cifra fijada por el Consejo de Ministros a través del Real Decreto 1462/2018 de 21 de Diciembre de 2018 


\section{Sugerencias}

\subsection{Fortalecer la enseñanza de lengua y cultura hispánicas y la optimización del currículo disciplinar, y mejorar las competencias interculturales}

Como se ha mencionado arriba, las diferencias de docencia y evaluación entre China y España hacen que los estudiantes de intercambio se adapten con dificultad a los estudios en el país de acogida, lo cual afecta directamente a la eficiencia y la evaluación de los programas de movilidad. Así que nos hacemos reflexionar sobre la enseñanza del español en China. En base de las características chinas y universitarias de la enseñanza de lenguas extranjeras (por ejemplo, los elementos de la cultura vernácula en el aula de ELE) se debería introducir el Marco Europeo de Referencia para las Lenguas, reintegrar el currículo disciplinar, reformar y perfeccionar los contenidos de asignaturas enfatizando la capacitación y entrenamiento de las habilidades profesionales básicas de español, y al mismo que se conservan las ventajas del método tradicional de enseñanza «traducción y gramática» se aplica el método de comunicación en el aula ELE. Además, se debería añadir asignaturas como educación internacional, comunicación intercultural para integrar los conocimientos sociales y culturales relevantes de los países de habla hispana para que los alumnos aprendan a resolver por sí mismos obstáculos, barreras y choques culturales, de manera que se promueven mejor los intercambios y cooperación internacionales.

\subsection{Implementar la formación previa a la partida y establecer la plataforma de información y comunicación}

Las vacantes de plataformas de información y comunicación hacen que los esfuerzos por la cooperación internacional carezcan de eficiencia y motivación. Tres tipos de plataformas serían necesarios:

\section{·Plataforma interuniversitaria \\ ·Plataforma universidad-alumnado \\ ·Plataforma inter-alumnado}

En la plataforma interuniversitaria se intercambian informaciones: presentación de la universidad y de los departamentos, currículo disciplinar, calendario académico, documentos y requisitos para la matrícula y la visa, etc.

En la plataforma universidad-alumnado se publican informaciones: presentación de los programas de movilidad internacional, fechas de entrega de la solicitud, canales de financiamiento, mecanismo de selección, formaciones orientativas, servicios prestados, normas del reconocimiento de créditos, etc. Y la universidad también puede mantenerse informada del proceso de solicitud del alumnado y de su proceso de intercambio en España.

Y la plataforma inter-alumnado es de carácter más como sala de charla, en la que los alumnos se intercambian las informaciones obtenidas, se resuelven problemas y dudas y comparten experiencias. 


\subsection{Aumentar la financiación y mejorar el mecanismo de becas y ayudas económicas}

Tanto las universidades españolas como chinas deberían hacer todo lo posible para ampliar recursos financieros, estandarizar el proceso de la solicitud para que los alumnos de condiciones económicas bajas tengan la oportunidad de sacar a provecho, de manera equitativa, los programas de movilidad. Los gobiernos, las empresas, los antiguos alumnos son canales potenciales de la financiación.

\section{Conclusiones}

La movilidad internacional de estudiantes es considerada como un instrumento de cooperación, presidida por consideraciones político- culturales, de fomento del intercambio académico o de ayuda al desarrollo, la cual es también uno de los canales importantes de conocimiento mutuo entre países y culturas diferentes. Y los programas políticos y académicos inspirados en este enfoque han tenido un papel muy importante a lo largo de la historia.

La internacionalización es el objetivo y la característica que aspiran las universidades tanto europeas como chinas. Los estudiantes son los principales beneficiarios de los programas de movilidad internacional. El aprendizaje centrado en el alumno y la movilidad les ayudarán a desarrollar las competencias que necesitan en un mercado laboral cambiante y les facultarán para convertirse en ciudadanos activos y responsables.

Con la no larga existencia de la especialidad universitaria de español en China, los programas de movilidad de sus estudiantes están experimentando un desarrollo rápido, y han sido altamente evaluados según el resultado del cuestionario de este estudio. Pero también es un desarrollo defectuoso, que reside principalmente en la falta de control de calidad. A pesar de que esto se debe en mayor parte a los recursos humanos y la experiencia limitados, reconocemos que algunas mejoras son posibles de realizar si somos conscientes de que las necesidades del alumno siempre son el punto de partida de las políticas educativas. Guiadas por esta ideología, las universidades deberían tomar estrategias antes, durante y después de la puesta en marcha de los programas de la movilidad estudiantil, las cuales incluyen pero no se limitan al diseño del plan curricular, orientación al escoger asignaturas, capacitación de la competencia intercultural durante todo el proceso de enseñanzaaprendizaje, establecimiento y mantenimiento de plataformas de comunicación, mejorar la gestión por procesos y el sistema de evaluación, entre muchas otras.

\section{Referencias bibliográficas}

Agencia Nacional de Evaluación de la Calidad y Acreditación (2009). El Comunicado de Lovaina-la Nueva. Sitio web: http://www.aneca.es/Actividad-internacional/ Documentos-internacionales-de-referencia/Comunicados-de-los-ministroseuropeos [Consulta: 20/06/2019].

Cordera Campos, R., \& Santamaría Ambriz, R. (2008). Internacionalización, autonomía y calidad de la educación superior: Elementos para la integración de América Latina y el Caribe. Universidades, 58(37), 69-76. 
Declaración de Bolonia (1999). Declaración conjunta de los Ministros Europeos de Educación. Sitio web: http://www.eees.es/pdf/Bolonia_ES.pdf [Consulta: 20/06/2019].

Luchilo, L. (2006). Movilidad de estudiantes universitarios e internacionalización de la educación superior. CTS: Revista iberoamericana de ciencia, tecnología y sociedad, 3(7), 105-133.

Ministerio de Educación chino.(2017). Plan Nacional de Reforma y Desarrollo dela Educación a Mediano y Largo Plazo. Sitio web: http://old.moe.gov.cn/publicfiles/business/ htmlfiles/moe/info_list/201407/xxgk_171904.html [Consulta: 20/06/2019].

Palma, J. J. G. (2013). Movilidad estudiantil internacional y cooperación educativa en el nivel superior de educación 1. Revista Iberoamericana de Educación, 61, 59-76.

吴剑桥. (2019). 关于近十年中国高等教育国际化政策研究的趋势分析. 教育观察(10), 30-33.

Wu, J. Q. (2019). Análisis de la tendencia de la investigación sobre la política de internacionalización de la educación superior de China en los últimos diez años. Observación de la educación, 10, 30-33. 